\title{
Histidinol phosphate phosphatase of Mtb: Structural and functional analysis
}

Bhavya Jha ${ }^{1}$, Deepak Kumar ${ }^{1}$, Khundrakpam Herojit Singh ${ }^{1}$, Anam Ashraf ${ }^{1}$, Bichitra Kumar Biswal ${ }^{1}$

${ }^{1}$ Protein Crystallography Lab, National Institute Of Immunology, New Delhi, India E-mail: jhabhavya@nii.ac.in

The global tuberculosis (TB) epidemic, caused by the pathogen Mycobacterium tuberculosis (Mtb), is aggravated by the emergence of extremely drug resistant and multi-drug resistant strains. The present situation necessitates the identification and characterization of new drug targets. The histidine (His) biosynthetic pathway (Figure), which converts 5phosphoribosyl-1-pyrophosphate to histidine in ten enzymatic steps, is essential for Mtb growth but is absent in mammals, thus making it an attractive anti-TB drug target. The enzymes of the His pathway are largely conserved across the bacteria, fungi and lower eukaryotes that synthesize histidine de novo with some differences in a few catalytic steps; particularly the fifth, sixth and eighth steps. The determination of the Mtb genome sequence provided a map of the genes involved in its His pathway. However, it was unclear which particular enzyme catalyzes the eighth step- the dephosphorylation of Histidinol phosphate(HOLP) to Histidinol(HOL). In this study, employing bioinformatic approaches, the Histidinol phosphate phosphatase of Mtb was identified. The target protein was overexpressed in M. smegmatis and purified to homogenity. Its function was established using biochemical assays. The protein was crystallized in native as well as in substrate-bound complex forms and their structures were determined. Residues that line the active site pocket and the residues that are involved in catalysis were determined using both crystal structure and mutational studies data. The biological functional unit of His $\mathrm{N}$ is a dimer, the overall structure of a monomer (260 amino acids) folds into two distinct structural domains, $\mathrm{N}$ and $\mathrm{C}$ terminal domains. A loop of 20-residue long connects these two domains. The $\mathrm{N}$-terminal domain consists of residues 2130. Its tertiary structure comprised of two long alpha helices followed by a six-stranded anti-parallel $\beta$-sheet. The Cterminal domain comprised of residues 150-260 and folds into a globular structure consisting a five-stranded anti-parallel $\beta$ sheet sandwiched between six a-helices.

1. Sassetti, C. M. et al. (2003). Mol Microbiol., 48, 77-84.

2. Parish, T. (2003). Journal of Bacteriology, 185(22), 6702-6706.

3. Cole, S. T. et al. (1998). Nature, 393, 537-44.

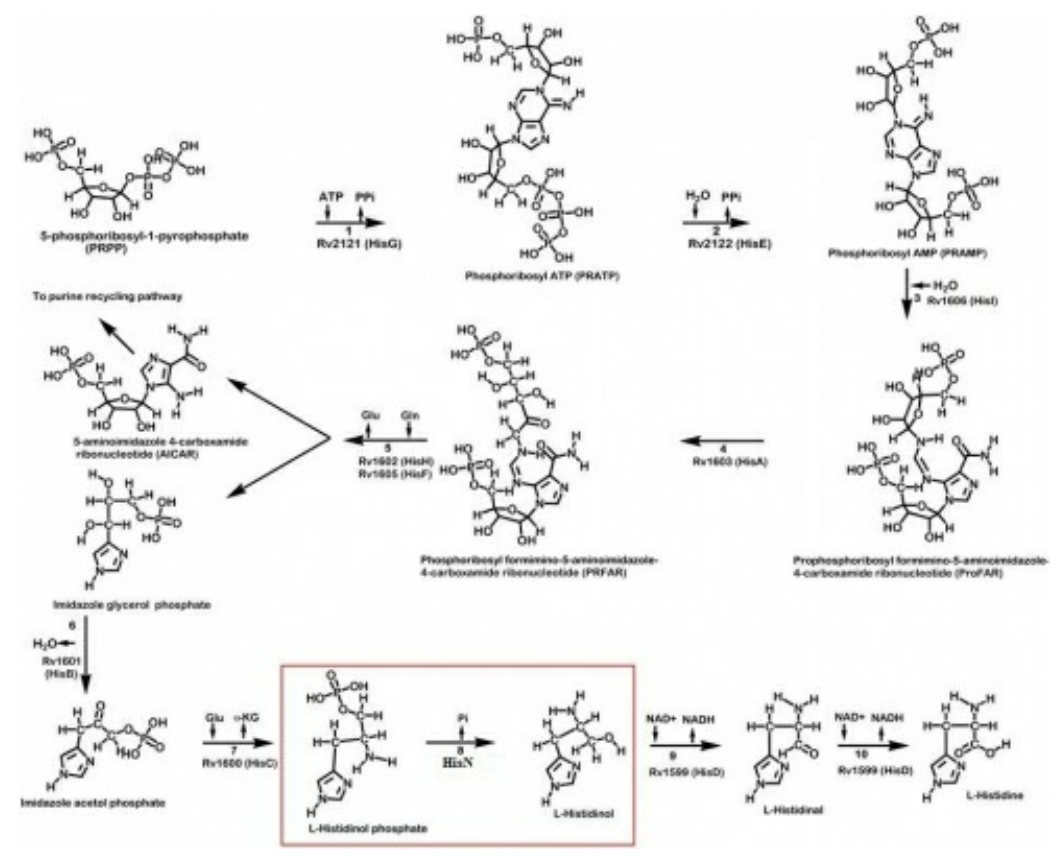

Keywords: Mycobacterium tuberculosis, Histidinol phosphate phosphatase 